Fuego. The species occurs in Patagonia and in the southern United States, but the birds from these two areas are specifically distinct.

The second erroneous statement is in the final paragraph of the same section, where (2) and (4) contradict each other :

"(2) ... a desert subspecies, $P$. $m$. sonoriensis, reared for eight years in a humid environment, is entirely unmodified in the direction of the humid subspecies $P$. m. gambeli.

(4) Similar results from transplantation of $P . \mathrm{m}$. rubidus and $P . m$. sonoriensis are obtained : reared in an entirely new environment, they do not converge toward each other but toward local humid subspecies P. m. gambeli."

Here (4) should read :

"Similar results from transplantation of $P$. $m$. rubidus and $P$. $m$. sonoriensis are obtained: reared in an entirely new environment, they do not converge toward each other or toward the local humid subspecies P. m. gambeli."

I regret having made these erroneous statements in a zoological field outside my own, and I trust that this correction will prevent their wider circulation.

Henry Fairfield Osborn.

American Museum of Natural History. September 2I.

The Spectrum of Zinc.

In the course of an investigation for the British Non-Ferrous Metals Research Association, which it is hoped will prove successful in applying the spectroscope to the quantitative assay of the impurities in zinc, the following observations of more special scientific interest were noted.

A zinc line, which does not appear to have been measured, occurs at $\lambda_{21} \mathrm{I}_{47} 36 \mathrm{I} . \AA .( \pm 0.02)$ near the cadmium line at $\lambda 2144.39 \AA$. Close to the "raie ultime ' of cadmium $\lambda_{2265^{\circ}} \mathrm{O}_{4} \AA$. is a zinc line the wavelength of which is $\lambda 2265.35$ I. $\AA$. $( \pm 0.02)$. This should be taken into account when searching the zinc spectrum for lines due to impurities, as with spectrographs of comparatively low dispersion, such as the Hilger quartz spectrograph size E. 3I., the zinc and cadmium lines are indistinguishable. Hagenbach and Schumacher (Z. wiss. Phot., 19, p. I29, I919) give the following as zinc lines: $\lambda \lambda 2265.08$ (8) and $2265^{\circ} 40(2)$, and it is highly probable that the former line is a cadmium line.

Spectrograms were taken on a Hilger quartz spectrograph size E. I. and the zinc lines measured on a photo-measuring micrometer size L. I. using various samples of zinc.

The samples of zinc supplied by the British NonFerrous Metals Research Association included one sample from the New Jersey Zinc Co., prepared by fractional distillation after electrolytic refining, and was considered to be spectroscopically pure.

D. M. SMITH.

Research Department, Adam Hilger, Ltd.,

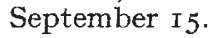

\section{Observed Relative Intensities of Stark Components in Hydrogen.}

ATtRACTED by the singularly large observed variations (Astrophys. Jour., 62, 229, I925) from estimated intensities of certain strong Stark components of $\mathrm{H}_{\beta}$ (H. A. Kramers, Copenhagen, I9I9) we have recently made quantitative measurements from a suitable source by means of a neutral wedge. It is interesting to compare the results with the new theoretical calculations by Schrödinger (Ann. d. Phys., 80,437, I926). Following are the observed, calculated, and estimated ratios of intensities of components having polarisations and displacements as indicated. The displacement $\Delta$ is expressed in the unit which appears in the quantum theory of the Stark-effect.

\begin{tabular}{|c|c|c|c|}
\hline Components Compared. & \multicolumn{3}{|c|}{ Ratio of Intensities. } \\
\hline $\begin{array}{l}p \text { components of } H_{\beta}, \frac{\Delta= \pm 8}{\Delta= \pm 10} \\
s \text { components of } H_{\beta}, \frac{\Delta= \pm 4}{\Delta= \pm 6}\end{array}$ & $\begin{array}{c}\text { Obs. } \\
\text { I } \cdot \text { oo }\end{array}$ & $\begin{array}{l}\text { Calc. } \\
\mathrm{I} \cdot 06 \\
\mathrm{I} \cdot 55\end{array}$ & $\begin{array}{l}\text { Est. } \\
0.40 \\
0.54\end{array}$ \\
\hline
\end{tabular}

The much weaker components, $p, \Delta= \pm 6$ and $s, \Delta= \pm 2$ appear somewhat stronger than the theory indicates. This is possibly due in part to superimposed secondary spectrum lines, which could not be detected by the present method. Owing to the increased interest in this research, the measurements are now being extended to the weaker components and to $\mathrm{H}_{\gamma}$ and $\mathrm{H}_{\delta}$ with such modification in method as to permit the detection of any seconday spectrum lines.

$$
\begin{aligned}
& \text { J. Stuart Foster. } \\
& \text { M. Laura Chalk. }
\end{aligned}
$$

(National Research Student.)

\section{McGill University, Montreal,} September 6.

\section{Absorption Spectrum of the Hydrogen Molecule.}

WiTH the continuous spectrum described by Lyman (Astrophysical Jour., 6o, I) as a background, and with hydrogen flowing through the receiver of the spectrograph, it was found possible to photograph the absorption spectrum of molecular hydrogen in the extreme ultra-violet. More than twenty absorption bands were observed between $\lambda \lambda_{I} 245$ and 840 . Most of the bands were clearly resolved and show the characteristic structure of the emission bands in the ultra-violet. The most striking feature of the spectrum is a progression of strong absorption bands beginning with $\lambda x$ I05. At least twelve bands of this progression were easily recognised. This adds a new electronic level to the three already obtained (Dieke and Hopfield, Oakland Meeting of the American Physical Society, June 1926) from the hydrogen emission spectrum. A continuous absorption spectrum begins sharply at $\lambda 840$. This continuous absorption corresponds to the dissociation of the molecule into a normal and an excited atom. The long wave-length limit of the continuous absorption compares well with the theoretical value 14.4 volts. Complete details will be published later.

\section{University of California,} Berkeley, August I7.

\section{Sterility in the Vegetable Marrow.}

THE vegetable marrow appears to have been affected by a form of sterility this season. I have been told of cases both in Gloucestershire and Surrey where the plants have produced an enormous number of male flowers and only one, or possibly two, female flowers. In the case of one marrow to a plant the resulting fruit has been a large one. Every plant was affected in the same way. No disbudding had been practised. In my own case I have had, as usual, an abundant crop, including about three dozen well-ripened fruits for winter use. The season has been favourable for half-hardy plants. Can any reader suggest a reason for this occurrence in a monœcious plant? ELEONORA ARMITAGE.

Dadnor, Herefordshire, September 25. 